\title{
新型腙基取代嘧啶衍生物的合成及抗肿瘤活性评价
}

\author{
张 洋 $a, b$ 张路野 $a, b$ \\ 刘丽敏 $a, b$ \\ 王 涛 $a, b$ \\ 孟娅琪 $a, b$ \\ 栗 娜 $a, b$ \\ 李二冬 $a, b$ \\ 汪正捷 $a, b$ \\ 刘秀娟 $a, b$ \\ 郑甲信 $a, c$ \\ 单丽红*, $a, b, c, d$ \\ 刘宏民*,a,b,c,d,e \\ 张秋荣*,a,b,c,d \\ $\left({ }^{a}\right.$ 郑州大学药学院 郑州 450001) \\ ( ${ }^{b}$ 新药创制与药物安全性评价河南省协同创新中心 郑州 450001)

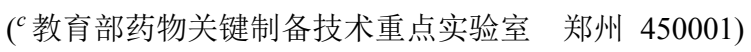 \\ ( ${ }^{d}$ 河南省药品质量与评价重点实验室 郑州 450001) \\ ( e 省部共建食管癌防治国家重点实验室 郑州 450052)
}

\begin{abstract}
摘要 为了寻找高效的新型抗肿瘤药物, 设计并合成了一系列新型腙基取代的 2,4,6-取代嘧啶衍生物, 并对目标化合物 在 MCF-7(人乳腺癌细胞), MGC-803(人胃癌细胞系), PC-3(人前列腺癌细胞), Hela(人宫颈癌细胞)和 A549(人肺癌细胞) 进行抗肿瘤活性评价. 结果显示部分化合物对 PC-3 表现出中度至强效的抗肿瘤活性. 其中 2-(丙-2-炔-1-基硫 基)-4-(2-(吡啶-2-基亚甲基)-肼基)-6-(三氟甲基)嘧啶(12I)对 PC-3 具有较强的抗增殖活性, $\mathrm{IC}_{50}$ 为 $1.37 \mu \mathrm{mol} \cdot \mathrm{L}^{-1}$, 抗肿瘤 活性明显优于阳性对照药 5-氟尿嘧啶, 为抗肿瘤药物的研究提供了新的思路.
\end{abstract}

关键词 腙; 嘧啶衍生物; 合成; 抗肿瘤活性

\section{Synthesis and Antitumor Activity Evaluation of Novel Hydrazone- Substituted Pyrimidine Derivatives}

\author{
Zhang, Yang ${ }^{a, b}$ Zhang, Luye ${ }^{a, b}$ Liu, Limin ${ }^{a, b} \quad$ Wang, Tao ${ }^{a, b} \quad$ Meng, Yaqi ${ }^{a, b}$ \\ Li, Na ${ }^{a, b}$ Li, Erdong ${ }^{a, b} \quad$ Wang, Zhengjie ${ }^{a, b} \quad$ Liu, Xiujuan ${ }^{a, b} \quad$ Zheng, Jiaxin ${ }^{a, c}$

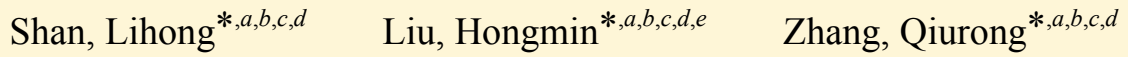 \\ ( ${ }^{a}$ School of Pharmaceutical Sciences, Zhengzhou University, Zhengzhou 450001) \\ ( ${ }^{b}$ Collaborative Innovation Center of New Drug Research and Safety Evaluation, Zhengzhou 450001) \\ ( ${ }^{c}$ Key Laboratory of Advanced Drug Preparation Technologies, Ministry of Education, Zhengzhou 450001) \\ ( ${ }^{d}$ Key Laboratory of Henan Province for Drug Quality and Evaluation, Zhengzhou 450001) \\ ( ${ }^{e}$ State Key Laboratory of Esophageal Cancer Prevention \& Treatment, Zhengzhou University, Zhengzhou 450052)
}

\begin{abstract}
In order to find more effective antitumor drugs, a series of novel hydrazone-substituted pyrimidine derivatives were designed, synthesized and evaluated for their antitumor activity against five different human cancer cell lines including MCF-7 (human breast cancer cell), MGC-803 (human gastric cancer cell), PC-3 (human prostate cancer cell), Hela (human cervical cancer cell) and A549 (human lung cancer cell) using methyl thiazolyl tetrazolium (MTT) assay. Most of the target compounds showed moderate to potent antitumor activity against five selected cancer cell lines. Among them, 2-(prop-2-yn-1-ylthio)-4(2-(pyridin-2-ylmethylene)-hydrazinyl)-6-(trifluoromethyl)pyrimidine (12I) displayed the most potent anti-proliferative activity against $\mathrm{PC}-3$ cell line $\left(\mathrm{IC}_{50}=1.37 \mu \mathrm{mol} \cdot \mathrm{L}^{-1}\right)$, which was significantly better than the positive control drug 5fluorouracil. This work provided clues to discover new antitumor agents.
\end{abstract}

Keywords hydrazone; pyrimidine derivatives; synthesis; antitumor activity

*Corresponding authors. E-mail: shlh@zzu.edu.cn; liuhm@zzu.edu.cn; zqr409@yeah.net

Received January 31, 2020; revised March 21, 2020; published online April 10, 2020.

Project supported by the National Natural Science Foundation of China (No. 81773562) and the National Key Research Program of Proteins (No. 2018YFE0195100) and the Openning Fund from State Key Laboratory of Esophageal Cancer Prevention \& Treatment (No. K2020000X).

国家自然科学基金(No. 81773562)、国家蛋白质研究项目(No. 2018YFE0195100)、省部共建食管癌防治国家重点实验室资助的开放基金(No. K2020000X)资助项目. 


\section{Introduction}

Cancer is still a leading life-threatening disease worldwide. The incidence and mortality rates of cancer in China account to $23.7 \%$ and $30 \%$, respectively, across the globe. ${ }^{[1,2]}$ This figure will further rise because of aging, intensification of industrialization and urbanization, lifestyle modifications, etc. Thus, the burden of cancer cannot be ignored. Although chemotherapy has emerged as one of the main strategies for the treatment of tumors, numerous drugs have produced multi-drug resistance and fatal side effects in clinical. ${ }^{[3]}$ As a consequence, it is of great significance to develop effective and side-effect anticancer drugs in the face of global cancer problems. Pyrimidine skeleton and heterocyclic annulated pyrimidines, elemental structural motifs of several synthetic and natural occurring products, attract great attention due to their remarkable biological activities such as antitumor, ${ }^{[4,5]}$ anti-inflammatory, ${ }^{[6]}$ anti-hepatitis, ${ }^{[7]}$ anti-diabetic ${ }^{[8]}$ and antimicrobial ${ }^{[9]}$ etc. For example, trifluorothymidine (1) can be used as a potential chemotherapeutic agent for the treatment of brain tumors, ${ }^{[10]}$ compound $\mathbf{2}$ is a potent and orally available agent of hedgehog signaling pathway inhibitor with improved physicochemical and pharmacokinetic properties, ${ }^{[11]}$ and compound $\mathbf{3}$ has promising activity against Hela cell line $\left(\mathrm{IC}_{50}=2.22 \mu \mathrm{mol} / \mathrm{L}\right)($ Figure 1$){ }^{[12]}$

Besides, hydrazine derivatives have recently received considerable attention on account of their diverse biological activities including antifungal, ${ }^{[13]}$ anti-bacterial, ${ }^{[14]}$ anti-inflammatory, anti-ulcerogenic, ${ }^{[15]}$ antiviral, ${ }^{[16,17]}$ antioxidant, ${ }^{[18]}$ antitumor ${ }^{[19]}$ and so on. ${ }^{[20]}$ Therefore, hydrazine as an active building block has important value and research significance in the design and synthesis of antitumor drugs. With the aim of identifying new antitumor agents, a series of novel 2,4,6-substituted pyrimidine derivatives based on molecular hybridization strategy were synthesized and evaluated for their antitumor activities.

\section{Results and discussion}

\subsection{Chemistry}

The synthetic strategy for the preparation of the target compound was depicted in Scheme 1. Firstly, compound 8 was prepared via cyclization reaction of ethyl 4,4,4-trifluoroacetoacetate with thiourea in the presence of potassium hydroxide under reflux in ethanol for $3 \mathrm{~h}$. Next, compound 8 and bromopropyne were reacted in $N, N$-dimethylformamide (DMF) at $90{ }^{\circ} \mathrm{C}$ for $4 \mathrm{~h}$ to obtain compound 9 . Then compound 9 was added to phosphorus oxychloride and then heated to $90{ }^{\circ} \mathrm{C}$ for $3 \mathrm{~h}$ to yield compound 10. Compound $\mathbf{1 0}$ was reacted with hydrazine hydrate in anhydrous ethanol at $80{ }^{\circ} \mathrm{C}$ for $2 \mathrm{~h}$ to obtain compound 11. Finally, compound 11 with appropriate aromatic aldehyde reacted in anhydrous ethanol at $80{ }^{\circ} \mathrm{C}$ for $3 \sim 6 \mathrm{~h}$ to obtain the

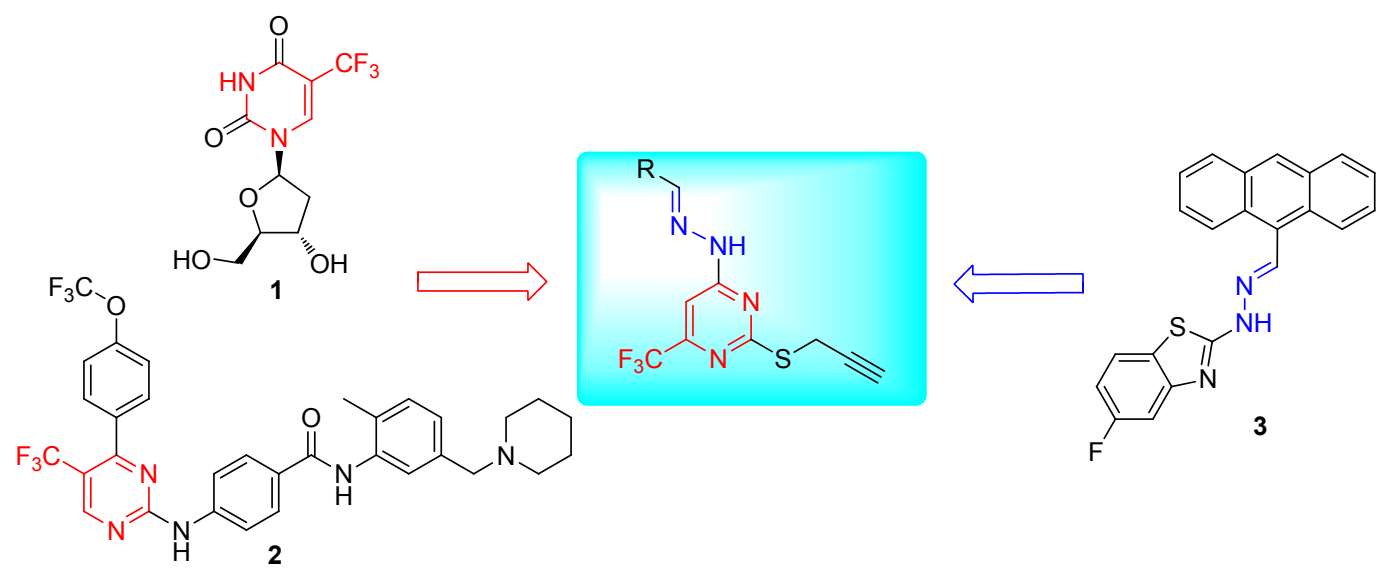

Figure 1 Representative anti-tumor agents and design of 2,4,6-substituted pyrimidines

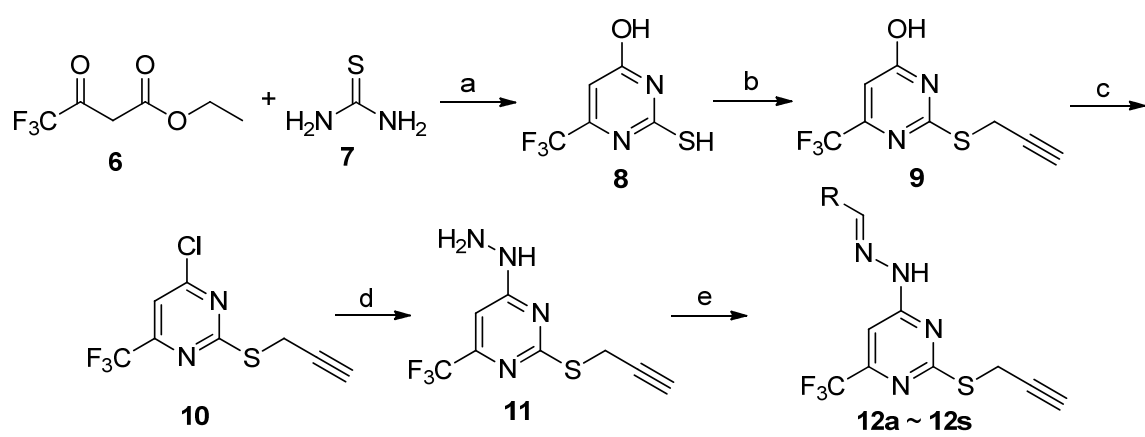

Reagents and conditions: (a) $\mathrm{CH}_{3} \mathrm{CH}_{2} \mathrm{OH}, \mathrm{KOH}, 80{ }^{\circ} \mathrm{C}, 3 \mathrm{~h}$; (b) $\mathrm{N}, \mathrm{N}$-dimethylformamide, $90{ }^{\circ} \mathrm{C}, 4 \mathrm{~h}$; (c) $\mathrm{POCl}_{3}, 90{ }^{\circ} \mathrm{C}, 3 \mathrm{~h}$; (d) $\mathrm{CH}_{3} \mathrm{CH}_{2} \mathrm{OH}$, hydrazine hydrate, $80{ }^{\circ} \mathrm{C}, 2 \mathrm{~h}$; (e) $\mathrm{CH}_{3} \mathrm{CH}_{2} \mathrm{OH}$, substituted benzaldehyde, $80{ }^{\circ} \mathrm{C}, 3 \sim 6 \mathrm{~h}$

Scheme 1 Synthesis of hydrazone-substituted pyrimidine derivatives 
target compounds $12 \mathrm{a} \sim \mathbf{1 2 0}$. The structures of compounds $12 \mathrm{a} \sim 120$ were fully characterized by ${ }^{1} \mathrm{H} N M R,{ }^{13} \mathrm{C} N M R$ and HRMS.

\subsection{Cytotoxic activity}

The anti-proliferative activities of the target compounds were evaluated against human cancer cell lines of different origins including MCF-7 (human breast cancer cell), MGC-803 (human gastric cancer cell), PC-3 (human prostate cancer cell), Hela (human cervical cancer cell) and A549 (human lung cancer cell) via the methyl thiazolyl tetrazolium (MTT) assay. 5-Fluorouracil (5-Fu) was employed as the positive drug. The results of preliminary biological evaluation were summarized in Table 1.

From the preliminary results in Table 1, the anti-proliferative activities of the intermediates 9, 10 and 11 possessed relatively weak anti-proliferative activity against the tested five cell lines. However, after a series of structural modifications, the anti-proliferative activities of the target compounds 12a $\sim \mathbf{1 2 0}$ were obviously improved, especially for PC-3. With the anti-proliferative activities against the cancer cell lines in hand, next, the preliminary structure-activity relationships of the target compounds were explored. Firstly, compared with compounds $12 \mathbf{b} \sim \mathbf{1 2 d}$, when the same electron-withdrawing group was introduced on the benzene ring, the anti-proliferative activities of the para-position against MCF-7, Hela and A549 were relatively better than the ortho and meta-positions. But the introduction of multiple electron-withdrawing groups such as compound 12e had little effect on the improvement of anti-proliferative activity. However, compared with compounds $\mathbf{1 2 f} \sim \mathbf{1 2} \mathbf{j}$, when an electron-donating group was introduced on the benzene ring, different sites and the number of substituents had greater influence on the antiproliferative activities, such as compounds $12 \mathbf{h} \sim \mathbf{1 2} \mathbf{j}$ contained electron-donating methoxy substituent, the antiproliferative activity of the meta-position except MCF-7 was relatively better than the ortho and para-positions. When aromatic substituent containing hetero atom was introduced into the structure of the compound, its antiproliferative activities against MCF-7, MGC-803 and PC-3 were significantly improved, compared with the positive control drug 5-fluorouracil. With the volume of aromatic substituent increasing, the anti-proliferative activity of the target compounds had been significantly decreased, such as compound 120. The 18 compounds in this series had certain activities on several tested tumor cells, among which PC-3 had the best anti-proliferative activity. Among them, the anti-proliferative activities of compound 12I against MCF-7, MGC-803, PC-3, and A549 were 9.18, $3.63,1.37,7.85 \mu \mathrm{mol} / \mathrm{L}$, respectively, significantly better than 5-fluorouracil.

\section{Conclusion}

In summary, a series of novel hydrazone-substituted pyrimidine derivatives were designed, synthesized and evaluated for their anti-proliferative activity against MCF-7, MGC-803, PC-3, Hela and A549 human cancer cell lines. Most of the synthesized compounds showed moderate to potent activity against five selected cancer cell lines. Among them, compound 12l displayed the most potent anti-proliferative activity against PC-3 cell line. Taken together, these results suggested that compound $\mathbf{1 2 l}$ might be a valuable lead compound for anti-tumor agents.

Table 1 Antitumor activities $\left[\mathrm{IC}_{50} /\left(\mu \mathrm{mol} \cdot \mathrm{L}^{-1}\right)\right]$ of target compounds against five cancer cell lines

\begin{tabular}{|c|c|c|c|c|c|c|}
\hline Compd. & $\mathrm{R}$ & MCF-7 & MGC-803 & PC-3 & Hela & A549 \\
\hline 9 & - & $>64$ & $28.15 \pm 1.56$ & $>64$ & $>64$ & $>64$ \\
\hline 10 & - & $>64$ & $26.56 \pm 1.53$ & $>64$ & $>64$ & $>64$ \\
\hline 11 & - & $>64$ & $25.47 \pm 1.51$ & $>64$ & $42.43 \pm 1.62$ & $>64$ \\
\hline $12 a$ & $\mathrm{C}_{6} \mathrm{H}_{5}$ & $8.23 \pm 1.27$ & $11.38 \pm 1.40$ & $3.52 \pm 0.90$ & $27.87 \pm 1.44$ & $33.09 \pm 1.62$ \\
\hline $12 b$ & $2-\mathrm{ClC}_{6} \mathrm{H}_{4}$ & $11.63 \pm 1.03$ & $7.06 \pm 0.89$ & $8.61 \pm 1.31$ & $36.01 \pm 1.55$ & $30.43 \pm 1.59$ \\
\hline $12 \mathrm{c}$ & $3-\mathrm{ClC}_{6} \mathrm{H}_{4}$ & $12.80 \pm 1.45$ & $10.62 \pm 1.16$ & $3.82 \pm 0.87$ & $26.79 \pm 1.42$ & $46.55 \pm 1.77$ \\
\hline 12d & $4-\mathrm{ClC}_{6} \mathrm{H}_{4}$ & $8.21 \pm 1.04$ & $7.68 \pm 1.01$ & $4.21 \pm 0.83$ & $17.97 \pm 1.23$ & $16.61 \pm 1.32$ \\
\hline $12 \mathrm{e}$ & $3,4-\mathrm{F}_{2} \mathrm{C}_{6} \mathrm{H}_{3}$ & $12.73 \pm 1.32$ & $10.18 \pm 1.06$ & $6.72 \pm 1.07$ & $19.27 \pm 1.28$ & $48.07 \pm 1.78$ \\
\hline $12 \mathrm{f}$ & $2-\mathrm{CH}_{3} \mathrm{C}_{6} \mathrm{H}_{4}$ & $11.52 \pm 1.37$ & $11.47 \pm 1.46$ & $9.71 \pm 1.26$ & $33.68 \pm 1.52$ & $27.45 \pm 1.54$ \\
\hline $12 \mathrm{~g}$ & $4-\mathrm{CH}_{3} \mathrm{C}_{6} \mathrm{H}_{4}$ & $12.76 \pm 1.05$ & $10.61 \pm 1.38$ & $9.47 \pm 1.32$ & $42.04 \pm 1.62$ & $28.78 \pm 1.56$ \\
\hline $12 \mathrm{~h}$ & $2-\mathrm{CH}_{3} \mathrm{OC}_{6} \mathrm{H}_{4}$ & 14. $21 \pm 1.63$ & $31.43 \pm 1.62$ & $19.27 \pm 1.07$ & $>64$ & $>64$ \\
\hline $12 \mathrm{i}$ & $3-\mathrm{CH}_{3} \mathrm{OC}_{6} \mathrm{H}_{4}$ & $29.51 \pm 1.36$ & $20.53 \pm 1.34$ & $15.31 \pm 1.19$ & $22.44 \pm 1.35$ & $47.03 \pm 1.78$ \\
\hline $12 j$ & $4-\mathrm{CH}_{3} \mathrm{OC}_{6} \mathrm{H}_{4}$ & $43.81 \pm 1.56$ & $55.10 \pm 1.87$ & $30.46 \pm 1.48$ & $>64$ & $>64$ \\
\hline $12 \mathrm{k}$ & 2-Thienyl & $8.32 \pm 1.13$ & $9.11 \pm 1.03$ & $6.83 \pm 0.97$ & $16.40 \pm 1.25$ & $24.05 \pm 1.44$ \\
\hline 121 & 2-Pyridinyl & $9.18 \pm 1.32$ & $3.63 \pm 0.82$ & $1.37 \pm 0.31$ & $23.89 \pm 1.37$ & $7.85 \pm 1.00$ \\
\hline $12 \mathrm{~m}$ & 3-Pyridinyl & $11.33 \pm 1.35$ & $5.78 \pm 1.04$ & $9.66 \pm 1.42$ & $28.45 \pm 1.45$ & $49.15 \pm 1.80$ \\
\hline $12 n$ & 4-Pyridinyl & $13.14 \pm 1.54$ & $5.38 \pm 0.92$ & $7.27 \pm 1.17$ & $21.06 \pm 1.32$ & $53.37 \pm 1.83$ \\
\hline 120 & 4-[1,1'-Biphenyl] & $12.16 \pm 1.41$ & $10.23 \pm 1.07$ & $8.46 \pm 1.27$ & $39.05 \pm 1.59$ & $54.14 \pm 1.84$ \\
\hline $5-\mathrm{Fu}^{b}$ & - & $13.52 \pm 0.93$ & $12.82 \pm 0.71$ & $9.83 \pm 0.58$ & $12.19 \pm 1.26$ & $10.38 \pm 1.25$ \\
\hline
\end{tabular}

${ }^{a}$ Antitumor activity was assayed by exposure for $72 \mathrm{~h}$ to substances and expressed as concentration required to inhibit tumor cell proliferation by $50 \%$ (IC ${ }_{50}$ ). Dates are presented as the mean \pm SDs of three independent experiments. ${ }^{b}$ Positive control. 


\section{Experimental section}

\subsection{Materials}

Silicagel: Qingdao Kangyexin Medicinal Silicone Desiccant Company Limited. Column chromatography silica gel: Yantai Jiangyou Silicone Development Company Limited. Potassium hydroxide: Tianjin Yongda Chemical Reagent Company Limited; Anhydrous Ethanol: Tianjin Yongda Chemical Reagent Company Limited. N,N-Dimethylformamide: Tianjin Yongda Chemical Reagent Company Limited. All reagents and solvents were purchased from commercial sources and were used without further purification. Melting points were determined on an X-5 micro melting apparatus and are uncorrected. ${ }^{1} \mathrm{H}$ NMR and ${ }^{13} \mathrm{C}$ NMR spectra were measured using a DPX-DPX400 superconducting nuclear magnetic resonance instrument. Chemical shifts $(\delta)$ are given relative to TMS. High-resolution mass spectra were measured using a Waters-Micromass Q-TofMicro High Resolution Determination of tetragonal-flight time tandem mass spectrometer.

\subsection{MTT assay}

Cells in the logarithmic growth phase were seeded in 96-well plates at 3000 5000 cells per well. After the cells were cultured for $24 \mathrm{~h}$, different concentrations of compounds were treated for $72 \mathrm{~h}$, respectively. MTT (methyl thiazolyl tetrazolium, Solarbio) was added to each well at a final concentration of $0.5 \mathrm{mg} / \mathrm{mL}$. After $4 \mathrm{~h}$ in a $37{ }^{\circ} \mathrm{C}$ incubator, the medium was aspirated. $150 \mathrm{~mL}$ of dimethyl sulfoxide (DMSO) was then added to each well to dissolve the formazan, and the plate was shaken on a shaker for 10 min. The absorbance was measured by an enzyme-linked immunosorbent assay reader (BioTek, USA) at a wavelength of $490 \mathrm{~nm}$, and the cell survival rate was measured. The concentration-response curve generated by SPSS 16.0 software was used to determine the concentration of compound $\left(\mathrm{IC}_{50}\right)$ required to inhibit $50 \%$ of cell growth. Results were Mean $\pm \mathrm{SD}$ of three independent experiments.

\subsection{Chemistry}

2-Mercapto-6-(trifluoromethyl)pyrimidin-4-ol (8), ${ }^{[21]}$ 2(prop-2-yn-1-ylthio)-6-(trifluoromethyl)pyrimidin-4-ol (9), ${ }^{[22]}$ 4-chloro-2-(prop-2-yn-1-ylthio)-6-(trifluoromethyl)pyrimidine $(\mathbf{1 0})^{[22]}$ were synthesized according to the literatures.

4.3.1 Synthesis of 4-hydrazinyl-2-(prop-2-yn-1-ylthio)-6-(trifluromethyl)pyrimidine (11)

Compound $10(2.11 \mathrm{~g}, 8.42 \mathrm{mmol})$ was added to $20 \mathrm{~mL}$ of absolute ethanol at room temperature, heated to $80{ }^{\circ} \mathrm{C}$, and hydrazine hydrate $(821 \mu \mathrm{L}, 16.84 \mathrm{mmol})$ was slowly added. The reaction was completed by thin-layer chromatography (TLC). The reaction mixture was cooled to room temperature and filtered to obtain 4-hydrazinyl-2-(prop2-yn-1-ylthio)-6-(trifluoromethyl)pyrimidine (11), white solid, yield 81.7\%. m.p. 123.1 124.6 ${ }^{\circ} \mathrm{C} ;{ }^{1} \mathrm{H}$ NMR (400 $\left.\mathrm{MHz}, \mathrm{DMSO}-d_{6}\right) \delta: 9.30(\mathrm{~s}, 1 \mathrm{H}), 6.93(\mathrm{~s}, 1 \mathrm{H}), 4.68(\mathrm{~s}, 2 \mathrm{H})$, $3.94(\mathrm{~d}, J=2.5 \mathrm{~Hz}, 2 \mathrm{H}), 3.12(\mathrm{~s}, 1 \mathrm{H}) ;{ }^{13} \mathrm{C} \mathrm{NMR}(101 \mathrm{MHz}$, DMSO- $\left.d_{6}\right) \delta: 170.9,168.7,121.5,104.5,74.2,73.2,33.5$;
HRMS (ESI) calcd for $\mathrm{C}_{8} \mathrm{H}_{8} \mathrm{~F}_{3} \mathrm{~N}_{4} \mathrm{~S}[\mathrm{M}+\mathrm{H}]^{+}$249.0422, found 249.0424 .

4.3.2 General procedure for the synthesis of compounds 12a $\sim 120$

Compound 11 (0.15 g, $0.609 \mathrm{mmol})$ was added in $6 \mathrm{~mL}$ of absolute ethanol at room temperature, heated to $80{ }^{\circ} \mathrm{C}$, and benzaldehyde $(70.44 \mu \mathrm{L}, 0.609 \mathrm{mmol})$ was added slowly. The reaction was completed by TLC, cooled to room temperature, and the mixture was stirred. Column chromatography $[V($ petroleum ether $): V($ ethyl acetate $)=$ $6: 1)$ gave compound 12a.

4-(2-Benzylidenehydrazinyl)-2-(prop-2-yn-1-ylthio)-6(trifluoromethyl)pyrimidine (12a): White solid, yield 52.5\%. m.p. $156.1 \sim 156.7{ }^{\circ} \mathrm{C} ;{ }^{1} \mathrm{H}$ NMR $(400 \mathrm{MHz}$, DMSO- $\left.d_{6}\right) \delta: 12.19(\mathrm{~s}, 1 \mathrm{H}), 8.23(\mathrm{~s}, 1 \mathrm{H}), 7.97(\mathrm{~d}, J=7.3$ $\mathrm{Hz}, 1 \mathrm{H}), 7.80 \sim 7.74(\mathrm{~m}, 2 \mathrm{H}), 7.46(\mathrm{~d}, J=6.4 \mathrm{~Hz}, 2 \mathrm{H})$, $7.27(\mathrm{~s}, 1 \mathrm{H}), 4.00(\mathrm{~d}, J=2.4 \mathrm{~Hz}, 2 \mathrm{H}), 3.19(\mathrm{t}, J=2.4 \mathrm{~Hz}$, $1 \mathrm{H}) ;{ }^{13} \mathrm{C}$ NMR $\left(101 \mathrm{MHz}, \mathrm{DMSO}-d_{6}\right) \delta: 169.9,167.3$, $162.0,145.8,133.8,132.8,130.1,129.2,128.8,95.9,79.9$, 73.4, 18.6; HRMS (ESI) calcd for $\mathrm{C}_{15} \mathrm{H}_{12} \mathrm{~F}_{3} \mathrm{~N}_{4} \mathrm{~S}[\mathrm{M}+\mathrm{H}]^{+}$ 337.0735 , found 337.0734 .

4-(2-(2-Chlorobenzylidene)hydrazinyl)-2-(prop-2-yn-1ylthio)-6-(trifluoromethyl)pyrimidine (12b): White solid powder, yield $62.7 \%$. m.p. $143.7 \sim 144.8{ }^{\circ} \mathrm{C} ;{ }^{1} \mathrm{H}$ NMR $\left(400 \mathrm{MHz}, \mathrm{DMSO}-d_{6}\right) \delta: 12.33(\mathrm{~s}, 1 \mathrm{H}), 8.62(\mathrm{~s}, 1 \mathrm{H})$, $8.13 \sim 8.05(\mathrm{~m}, 1 \mathrm{H}), 7.52 \sim 7.46(\mathrm{~m}, 1 \mathrm{H}), 7.46 \sim 7.39(\mathrm{~m}$, 2H), $7.27(\mathrm{~s}, 1 \mathrm{H}), 4.00(\mathrm{~d}, J=2.5 \mathrm{~Hz}, 2 \mathrm{H}), 3.19(\mathrm{t}, J=2.5$ $\mathrm{Hz}, 1 \mathrm{H}) ;{ }^{13} \mathrm{C}$ NMR $\left(101 \mathrm{MHz}, \mathrm{DMSO}-d_{6}\right) \delta: 170.0,162.0$, $141.6,132.9,131.2,131.1,129.8,127.4,126.9,121.9$, 119.2, 96.0, 79.7, 73.4, 18.6. HRMS (ESI) calcd for $\mathrm{C}_{15} \mathrm{H}_{11} \mathrm{ClF}_{3} \mathrm{~N}_{4} \mathrm{~S}[\mathrm{M}+\mathrm{H}]^{+}$371.0345, found 371.0344.

4-(2-(3-Chlorobenzylidene)hydrazinyl)-2-(prop-2-yn-1ylthio)-6-(trifluoromethyl)pyrimidine (12c): White solid, yield 59.8\%. m.p. 149.5 150.9 ${ }^{\circ} \mathrm{C} ;{ }^{1} \mathrm{H}$ NMR (400 MHz, DMSO- $\left.d_{6}\right) \delta: 12.28(\mathrm{~s}, 1 \mathrm{H}), 8.19(\mathrm{~s}, 1 \mathrm{H}), 7.86(\mathrm{~s}, 1 \mathrm{H}), 7.74$ (s, 1H), 7.48 (d, $J=4.7 \mathrm{~Hz}, 2 \mathrm{H}), 7.34$ (s, 1H), 3.99 (d, $J=$ $2.5 \mathrm{~Hz}, 2 \mathrm{H}), 3.18(\mathrm{t}, J=2.5 \mathrm{~Hz}, 1 \mathrm{H}) ;{ }^{13} \mathrm{C}$ NMR $(101 \mathrm{MHz}$, DMSO- $\left.d_{6}\right) \delta: 169.9,162.1,144.2,136.1,133.7,130.7$, 129.7, 126.4, 125.7, 121.9, 119.2, 96.2, 79.9, 73.4, 18.6; HRMS (ESI) calcd for $\mathrm{C}_{15} \mathrm{H}_{11} \mathrm{ClF}_{3} \mathrm{~N}_{4} \mathrm{~S}[\mathrm{M}+\mathrm{H}]{ }^{+}$ 371.0345 , found 371.0344 .

4-(2-(4-Chlorobenzylidene)hydrazinyl)-2-(prop-2-yn-1ylthio)-6-(trifluoromethyl)pyrimidine (12d): White solid, yield $60.3 \%$. m.p. $143.5 \sim 143.8{ }^{\circ} \mathrm{C}$; ${ }^{1} \mathrm{H}$ NMR (400 MHz, DMSO- $\left.d_{6}\right) \delta: 12.11(\mathrm{~s}, 1 \mathrm{H}), 8.51(\mathrm{~s}, 1 \mathrm{H}), 7.86(\mathrm{~d}, J=7.6$ $\mathrm{Hz}, 1 \mathrm{H}), 7.31 \sim 7.25(\mathrm{~m}, 3 \mathrm{H}), 7.20(\mathrm{~s}, 1 \mathrm{H}), 3.99(\mathrm{~d}, J=2.5$ $\mathrm{Hz}, 2 \mathrm{H}), 3.19(\mathrm{t}, J=2.5 \mathrm{~Hz}, 1 \mathrm{H}) ;{ }^{13} \mathrm{C} \mathrm{NMR}(101 \mathrm{MHz}$, DMSO- $\left.d_{6}\right) \delta: 169.9,161.9,144.9,136.6,130.9,129.7$, 126.2, 121.9, 119.2, 79.9, 73.4, 18.6; HRMS (ESI) calcd for $\mathrm{C}_{15} \mathrm{H}_{11} \mathrm{ClF}_{3} \mathrm{~N}_{4} \mathrm{~S}[\mathrm{M}+\mathrm{H}]^{+}$371.0345, found 371.0344 .

4-(2-(3,4-Difluorobenzylidene)hydrazinyl)-2-(prop-2yn-1-ylthio)-6-(trifluoromethyl)pyrimidine (12e): White solid, yield 33.1\%. m.p. 153.1 153.8 ${ }^{\circ} \mathrm{C}$; ${ }^{1} \mathrm{H}$ NMR $(400$ $\left.\mathrm{MHz}, \mathrm{DMSO}-d_{6}\right) \delta: 12.26(\mathrm{~s}, 1 \mathrm{H}), 8.16(\mathrm{~s}, 1 \mathrm{H}), 7.94(\mathrm{~d}$, $J=8.3 \mathrm{~Hz}, 1 \mathrm{H}), 7.64 \sim 7.57(\mathrm{~m}, 1 \mathrm{H}), 7.51(\mathrm{dd}, J=10.3$, $8.4 \mathrm{~Hz}, 1 \mathrm{H}), 7.36$ (s, 1H), 3.98 (d, $J=2.6 \mathrm{~Hz}, 2 \mathrm{H}), 3.18$ (t, 
$\mathrm{J}=2.6 \mathrm{~Hz}, 1 \mathrm{H}) ;{ }^{13} \mathrm{C}$ NMR $\left(101 \mathrm{MHz}\right.$, DMSO- $\left.d_{6}\right) \delta: 169.9$, $161.9,159.8,151.0,149.2,143.0,124.4,119.1,118.0$, $116.5,115.4,96.2,80.1,73.7,18.5$; HRMS (ESI) calcd for $\mathrm{C}_{15} \mathrm{H}_{10} \mathrm{~F}_{5} \mathrm{~N}_{4} \mathrm{~S}[\mathrm{M}+\mathrm{H}]^{+}$373.0546, found 373.0547.

4-(2-(2-Methylbenzylidene)hydrazinyl)-2-(prop-2-yn-1ylthio)-6-(trifluoromethyl)pyrimidine (12f): White solid, yield 59.0\%. m.p. $158.2 \sim 158.6{ }^{\circ} \mathrm{C} ;{ }^{1} \mathrm{H}$ NMR $(400 \mathrm{MHz}$, DMSO- $\left.d_{6}\right) \delta: 12.14(\mathrm{~s}, 1 \mathrm{H}), 8.57(\mathrm{~s}, 1 \mathrm{H}), 7.94(\mathrm{dd}, J=7.7$, $1.4 \mathrm{~Hz}, 1 \mathrm{H}), 7.46 \sim 7.39(\mathrm{~m}, 1 \mathrm{H}), 7.25(\mathrm{~s}, 1 \mathrm{H}), 7.10(\mathrm{~d}, J=$ $8.2 \mathrm{~Hz}, 1 \mathrm{H}), 7.02$ (t, $J=7.5 \mathrm{~Hz}, 1 \mathrm{H}), 3.98(\mathrm{~d}, J=2.6 \mathrm{~Hz}$, $2 \mathrm{H}), 3.87$ (s, $3 \mathrm{H}), 3.18(\mathrm{t}, J=2.6 \mathrm{~Hz}, 1 \mathrm{H}) ;{ }^{13} \mathrm{C}$ NMR $(101$ MHz, DMSO- $\left.d_{6}\right) \delta$ : $169.9,161.9,157.6,153.3,141.4$, 131.6, 125.6, 121.8, 120.7, 119.2, 111.7, 95.9, 79.9, 73.4, 55.7, 18.6; HRMS (ESI) calcd for $\mathrm{C}_{16} \mathrm{H}_{15} \mathrm{~F}_{3} \mathrm{~N}_{4} \mathrm{OS}[\mathrm{M}+$ $\mathrm{OH}]^{-}$367.0840, found 367.0800 .

4-(2-(4-Methylbenzylidene)hydrazinyl)-2-(prop-2-yn-1ylthio)-6-(trifluoromethyl)pyrimidine (12g): White solid, yield $48.0 \%$. m.p. $152.7 \sim 153.4{ }^{\circ} \mathrm{C} ;{ }^{1} \mathrm{H}$ NMR $(400 \mathrm{MHz}$, DMSO- $\left.d_{6}\right) \delta: 12.12(\mathrm{~s}, 1 \mathrm{H}), 8.18(\mathrm{~s}, 1 \mathrm{H}), 7.65(\mathrm{~d}, J=8.1$ $\mathrm{Hz}, 2 \mathrm{H}), 7.28(\mathrm{~d}, J=6.7 \mathrm{~Hz}, 1 \mathrm{H}), 7.25(\mathrm{~d}, J=4.0 \mathrm{~Hz}, 2 \mathrm{H})$, $3.98(\mathrm{~d}, J=2.5 \mathrm{~Hz}, 2 \mathrm{H}), 3.35$ (s, $3 \mathrm{H}), 3.18(\mathrm{t}, J=2.6 \mathrm{~Hz}$, $1 \mathrm{H}) ;{ }^{13} \mathrm{C}$ NMR (101 MHz, DMSO- $\left.d_{6}\right) \delta: 169.9,161.9$, 153.6, 146.0, 140.0, 131.1, 129.4, 127.0, 122.0, 95.9, 79.9, 73.4, 21.0, 18.6; HRMS (ESI) calcd for $\mathrm{C}_{16} \mathrm{H}_{14} \mathrm{~F}_{3} \mathrm{~N}_{4} \mathrm{~S}$ $[\mathrm{M}+\mathrm{H}]^{+}$351.0891, found 351.0892.

4-(2-(2-Methoxybenzylidene)hydrazinyl)-2-(prop-2-yn1-ylthio)-6-(trifluoromethyl)pyrimidine (12h): White solid, yield 47.3\%. m.p.143.1 143.8 ${ }^{\circ} \mathrm{C}$; ${ }^{1} \mathrm{H}$ NMR $(400 \mathrm{MHz}$, DMSO- $\left.d_{6}\right) \delta: 12.15(\mathrm{~s}, 1 \mathrm{H}), 8.57(\mathrm{~s}, 1 \mathrm{H}), 7.95(\mathrm{dd}, J=7.7$, $1.3 \mathrm{~Hz}, 1 \mathrm{H}), 7.45 \sim 7.39(\mathrm{~m}, 1 \mathrm{H}), 7.25(\mathrm{~s}, 1 \mathrm{H}), 7.11(\mathrm{~d}, J=$ $8.2 \mathrm{~Hz}, 1 \mathrm{H}), 7.02(\mathrm{t}, J=7.5 \mathrm{~Hz}, 1 \mathrm{H}), 3.98(\mathrm{~d}, J=2.6 \mathrm{~Hz}$, $2 \mathrm{H}), 3.86(\mathrm{~s}, 3 \mathrm{H}), 3.18(\mathrm{t}, J=2.6 \mathrm{~Hz}, 1 \mathrm{H}) ;{ }^{13} \mathrm{C}$ NMR $(101$ MHz, DMSO- $\left.d_{6}\right) \delta$ : $169.9,161.9,157.6,153.5,141.5$, 131.6, 125.7, 121.9, 120.8, 119.2, 111.8, 95.9, 79.9, 73.4, 55.7, 18.6; HRMS (ESI) calcd for $\mathrm{C}_{16} \mathrm{H}_{14} \mathrm{~F}_{3} \mathrm{~N}_{4} \mathrm{OS}[\mathrm{M}+\mathrm{H}]^{+}$ 367.0840 , found 367.0841 .

4-(2-(3-Methoxybenzylidene)hydrazinyl)-2-(prop-2-yn1-ylthio)-6-(trifluoromethyl)pyrimidine (12i): White solid, yield $47.5 \%$. m.p. $147.8 \sim 148.3{ }^{\circ} \mathrm{C} ;{ }^{1} \mathrm{H}$ NMR $(400 \mathrm{MHz}$, DMSO- $\left.d_{6}\right) \delta: 12.18(\mathrm{~s}, 1 \mathrm{H}), 8.18(\mathrm{~s}, 1 \mathrm{H}), 7.37(\mathrm{~s}, 1 \mathrm{H}), 7.35$ (d, $J=2.5 \mathrm{~Hz}, 1 \mathrm{H}), 7.30(\mathrm{~s}, 1 \mathrm{H}), 7.27(\mathrm{~s}, 1 \mathrm{H}), 7.01$ (ddd, $J=7.7,2.5,1.6 \mathrm{~Hz}, 1 \mathrm{H}), 3.99(\mathrm{~d}, J=2.5 \mathrm{~Hz}, 2 \mathrm{H}), 3.85 \sim$ $3.80(\mathrm{~m}, 3 \mathrm{H}), 3.18(\mathrm{t}, J=2.6 \mathrm{~Hz}, 1 \mathrm{H}) ;{ }^{13} \mathrm{C}$ NMR $(101$ $\mathrm{MHz}$, DMSO- $\left.d_{6}\right) \delta$ : $170.1,161.9,159.5,145.7,135.2$, 129.9, 129.6, 121.9, 119.6, $116.0111 .8,96.0,79.973 .4$, 55.1, 18.6; HRMS (ESI) calcd for $\mathrm{C}_{16} \mathrm{H}_{14} \mathrm{~F}_{3} \mathrm{~N}_{4} \mathrm{OS}[\mathrm{M}+\mathrm{H}]^{+}$ 367.0840 , found 367.0842 .

4-(2-(4-Methoxybenzylidene)hydrazinyl)-2-(prop-2-yn1-ylthio)-6-(trifluoromethyl)pyrimidine (12j): White solid, yield $72.1 \%$. m.p. $138.9 \sim 140.1{ }^{\circ} \mathrm{C} ;{ }^{1} \mathrm{H}$ NMR $(400 \mathrm{MHz}$, DMSO- $\left.d_{6}\right) \delta: 12.07(\mathrm{~s}, 1 \mathrm{H}), 8.16(\mathrm{~s}, 1 \mathrm{H}), 7.71(\mathrm{~d}, J=8.8$ $\mathrm{Hz}, 2 \mathrm{H}), 7.23(\mathrm{~s}, 1 \mathrm{H}), 7.01(\mathrm{~d}, J=8.8 \mathrm{~Hz}, 2 \mathrm{H}), 3.98(\mathrm{~d}, J=$ $2.5 \mathrm{~Hz}, 2 \mathrm{H}), 3.81(\mathrm{~s}, 3 \mathrm{H}), 3.18(\mathrm{t}, J=2.6 \mathrm{~Hz}, 1 \mathrm{H}) ;{ }^{13} \mathrm{C}$ NMR (101 MHz, DMSO- $\left.d_{6}\right) \delta: 169.8,161.8,160.9145 .8$, 128.7, 126.4, 122.0, 119.2, 114.3, 95.8, 79.9, 73.4, 55.3, 18.5; HRMS (ESI) calcd for $\mathrm{C}_{16} \mathrm{H}_{14} \mathrm{~F}_{3} \mathrm{~N}_{4} \mathrm{OS}[\mathrm{M}+\mathrm{H}]^{+}$ 367.0840 , found 367.0839 .
2-(Prop-2-yn-1-ylthio)-4-(2-(thiophen-2-ylmethylene)hydrazinyl)-6-(trifluoromethyl)pyrimidine (12k): White solid, yield $70.2 \%$. m.p. $130.5 \sim 131.3{ }^{\circ} \mathrm{C} ;{ }^{1} \mathrm{H}$ NMR (400 MHz, DMSO- $\left.d_{6}\right) \delta: 12.19(\mathrm{~s}, 1 \mathrm{H}), 8.86(\mathrm{~s}, 1 \mathrm{H}), 8.40(\mathrm{~s}$, $1 \mathrm{H}), 7.68(\mathrm{~d}, J=5.0 \mathrm{~Hz}, 1 \mathrm{H}), 7.49(\mathrm{~d}, J=2.9 \mathrm{~Hz}, 1 \mathrm{H}), 7.06$ (s, $1 \mathrm{H}), 3.98(\mathrm{~d}, J=2.5 \mathrm{~Hz}, 2 \mathrm{H}), 3.18(\mathrm{t}, J=2.6 \mathrm{~Hz}, 1 \mathrm{H})$; ${ }^{13} \mathrm{C}$ NMR $\left(101 \mathrm{MHz}\right.$, DMSO- $\left.d_{6}\right) \delta: 170.0,161.6,155.7$, $140.9,138.5,133.8,131.0,130.8,129.0,128.0,79.9,73.5$, 18.5; HRMS (ESI) calcd for $\mathrm{C}_{13} \mathrm{H}_{10} \mathrm{~F}_{3} \mathrm{~N}_{4} \mathrm{~S}_{2}[\mathrm{M}+\mathrm{H}]^{+}$ 343.0299 , found 343.0300 .

2-(Prop-2-yn-1-ylthio)-4-(2-(pyridin-2-ylmethylene)hydrazinyl)-6-(trifluoromethyl)pyrimidine (121): White solid, yield 58.6\%. m.p. $195.8 \sim 196.6{ }^{\circ} \mathrm{C} ;{ }^{1} \mathrm{H}$ NMR (400 MHz, DMSO- $\left.d_{6}\right) \delta: 12.37(\mathrm{~s}, 1 \mathrm{H}), 8.61(\mathrm{~d}, J=4.7 \mathrm{~Hz}, 1 \mathrm{H})$, $8.25(\mathrm{~s}, 1 \mathrm{H}), 8.11(\mathrm{~d}, J=7.9 \mathrm{~Hz}, 1 \mathrm{H}), 7.88(\mathrm{dd}, J=10.9$, $4.5 \mathrm{~Hz}, 1 \mathrm{H}), 7.46 \sim 7.39(\mathrm{~m}, 1 \mathrm{H}), 7.35(\mathrm{~s}, 1 \mathrm{H}), 4.00(\mathrm{~d}, J=$ $2.5 \mathrm{~Hz}, 2 \mathrm{H}), 3.19(\mathrm{t}, J=2.5 \mathrm{~Hz}, 1 \mathrm{H}) ;{ }^{13} \mathrm{C}$ NMR $(101 \mathrm{MHz}$, DMSO- $\left.d_{6}\right) \delta: 170.1,162.2,159.7,152.7,149.5,146.4$, $146.1,136.8,124.3,120.2,96.2,79.9,73.5,20.2$, 18.6. HRMS (ESI) calcd for $\mathrm{C}_{14} \mathrm{H}_{11} \mathrm{~F}_{3} \mathrm{~N}_{5} \mathrm{~S}[\mathrm{M}+\mathrm{H}]^{+} 338.0687$, found 338.0686 .

2-(Prop-2-yn-1-ylthio)-4-(2-(pyridin-3-ylmethylene)hydrazinyl)-6-(trifluoromethyl)pyrimidine (12m): White solid, yield 59.4\%. m.p. $185.5 \sim 186.2{ }^{\circ} \mathrm{C} ;{ }^{1} \mathrm{H}$ NMR (400 MHz, DMSO- $\left.d_{6}\right) \delta: 12.34(\mathrm{~s}, 1 \mathrm{H}), 8.92(\mathrm{~s}, 1 \mathrm{H}), 8.61(\mathrm{~d}$, $J=4.6 \mathrm{~Hz}, 1 \mathrm{H}), 8.24(\mathrm{~d}, J=8.3 \mathrm{~Hz}, 2 \mathrm{H}), 7.48(\mathrm{dd}, J=7.8$, $4.6 \mathrm{~Hz}, 1 \mathrm{H}), 7.36(\mathrm{~s}, 1 \mathrm{H}), 3.99$ (d, $J=2.4 \mathrm{~Hz}, 2 \mathrm{H}), 3.19$ (d, $J=2.6 \mathrm{~Hz}, 1 \mathrm{H}) ;{ }^{13} \mathrm{C}$ NMR $\left(101 \mathrm{MHz}\right.$, DMSO- $\left.d_{6}\right) \delta: 170.0$, $162.2,150.6,148.7142 .9,136.9,133.5,129.8,123.9$, 121.9, 96.2, 79.9, 73.5, 18.6; HRMS (ESI) calcd for $\mathrm{C}_{14} \mathrm{H}_{11} \mathrm{~F}_{3} \mathrm{~N}_{5} \mathrm{~S}[\mathrm{M}+\mathrm{H}]^{+}$338.0687, found 338.0685.

2-(Prop-2-yn-1-ylthio)-4-(2-(pyridin-4-ylmethylene)hydrazinyl)-6-(trifluoromethyl)pyrimidine (12n): White solid, yield $72.1 \%$. m.p. $175.8 \sim 176.3{ }^{\circ} \mathrm{C} ;{ }^{1} \mathrm{H}$ NMR $(400$ MHz, DMSO- $\left.d_{6}\right) \delta: 12.44(\mathrm{~s}, 1 \mathrm{H}), 8.65(\mathrm{~d}, J=5.4 \mathrm{~Hz}, 2 \mathrm{H})$, $8.19(\mathrm{~s}, 1 \mathrm{H}), 7.74(\mathrm{~d}, J=5.9 \mathrm{~Hz}, 2 \mathrm{H}), 7.37(\mathrm{~s}, 1 \mathrm{H}), 4.00(\mathrm{~d}$, $J=2.5 \mathrm{~Hz}, 2 \mathrm{H}), 3.19(\mathrm{t}, J=2.6 \mathrm{~Hz}, 1 \mathrm{H}) ;{ }^{13} \mathrm{C}$ NMR $(101$ MHz, DMSO- $\left.d_{6}\right) \delta$ : $170.1,162.3,150.2,143.2,140.9$, $121.9,120.9,119.2,96.3,79.8,73.5,18.6$; HRMS (ESI) calcd for $\mathrm{C}_{14} \mathrm{H}_{11} \mathrm{~F}_{3} \mathrm{~N}_{5} \mathrm{~S}[\mathrm{M}+\mathrm{H}]^{+}$338.0687, found 338.0688 .

4-(2-([1,1'-Biphenyl]-4-ylmethylene)hydrazinyl)-2(prop-2-yn-1-ylthio)-6-(trifluoromethyl)pyrimidine (12o): White solid, yield 72.3\%. m.p. $150.2 \sim 150.9{ }^{\circ} \mathrm{C} ;{ }^{1} \mathrm{H}$ NMR $\left(400 \mathrm{MHz}, \mathrm{DMSO}-d_{6}\right) \delta: 12.23(\mathrm{~s}, 1 \mathrm{H}), 8.26(\mathrm{~s}, 1 \mathrm{H}), 7.86$ $(\mathrm{d}, J=8.3 \mathrm{~Hz}, 2 \mathrm{H}), 7.76(\mathrm{~d}, J=8.3 \mathrm{~Hz}, 2 \mathrm{H}), 7.72(\mathrm{~d}, J=$ $7.5 \mathrm{~Hz}, 2 \mathrm{H}), 7.50(\mathrm{t}, J=7.6 \mathrm{~Hz}, 2 \mathrm{H}), 7.40(\mathrm{t}, J=7.3 \mathrm{~Hz}$, $1 \mathrm{H}), 7.30(\mathrm{~s}, 1 \mathrm{H}), 3.99(\mathrm{~d}, J=2.4 \mathrm{~Hz}, 2 \mathrm{H}), 3.18(\mathrm{t}, J=2.5$ $\mathrm{Hz}, 1 \mathrm{H}) ;{ }^{13} \mathrm{C}$ NMR $\left(101 \mathrm{MHz}\right.$, DMSO- $\left.d_{6}\right) \delta: 192.6,169.8$, $161.9,145.3,141.4,139.1,132.6,130.3,128.9,127.5$, 126.9, 126.5, 119.3, 95.8, 79.8, 73.3, 18.4; HRMS (ESI) calcd for $\mathrm{C}_{21} \mathrm{H}_{16} \mathrm{~F}_{3} \mathrm{~N}_{4} \mathrm{~S}[\mathrm{M}+\mathrm{H}]^{+}$413.1048, found 413.1049 .

Supporting Information ${ }^{1} \mathrm{H}$ NMR and ${ }^{13} \mathrm{C}$ NMR spectra of compounds $9, \mathbf{1 0}$ and $\mathbf{1 1}$ as well as 12a $\sim 12 \mathrm{o}$ are available for free download from our website (http://sioc- 
journal.cn/).

\section{References}

[1] Bray, F.; Ferlay, J.; Soerjomataram, I.; Siegel, R. L.; Torre, L. A.; Jemal, A. CA Cancer J. Clin. 2018, 68, 394.

[2] Ferlay, J.; Colombet, M.; Soerjomataram, I.; Mathers, C.; Parkin, D. M.; Pineros, M.; Znaor, A.; Bray, F. Int. J. Cancer 2019, 144, 1941.

[3] Lavi, O.; Gottesman, M. M.; Levy, D. Drug Resist. Updates 2012, $15,90$.

[4] Li, E. D.; Lin, Q.; Meng, Y. Q.; Zhang, L. Y.; Song, P. P.; Li, N.; Xin, J. C.; Yang, P.; Bao, C. N.; Zhang, D. Q.; Zhang, Y.; Wang, J. K.; Zhang, Q. R.; Liu, H. M. Eur. J. Med. Chem. 2019, 172, 36.

[5] Meng, Y.; Li, E.; Zhang, Y.; Liu, S.; Bao, C.; Yang, P.; Zhang, L.; Zhang, D.; Wang, J.; Chen, Y.; Li, N.; Xin, J.; Zhao, P.; Ke, Y.; Zhang, Q.; Liu, H. Chin. J. Org. Chem 2019, 39, 2541 (in Chinese). (孟娅琪, 李二冬, 张洋, 刘栓, 包崇男, 杨鹏, 张路野, 张丹青, 王继宽, 陈雅欣, 栗娜, 辛景超, 赵培荣, 可钰, 张秋荣, 刘宏 民, 有机化学, 2019, 39, 2541.)

[6] Keche, A. P.; Hatnapure, G. D.; Tale, R. H.; Rodge, A. H.; Birajdar, S. S.; Kamble, V. M. Bioorg. Med. Chem. Lett. 2012, 22, 3445.

[7] Shakya, N.; Vedi, S.; Liang, C.; Yang, F.; Agrawal, B.; Kumar, R. Bioorg. Med. Chem. Lett. 2014, 24, 1407.

[8] Yang, Z.; Fang, Y.; Pham, T. A.; Lee, J.; Park, H. Bioorg. Med. Chem. Lett. 2013, 23, 1519.

[9] Mantipally, M.; Gangireddy, M. R.; Gundla, R.; Badavath, V. N.; Mandha, S. R.; Maddipati, V. C. Bioorg. Med. Chem. Lett. 2019, $29,2248$.
[10] Pouremad, R.; Bahk, K. D.; Shen, Y. J.; Knop, R. H.; Wyrwicz, A. M. NMR Biomed. 1999, 12, 373 .

[11] Xin, M.; Wen, J.; Tang, F.; Tu, C.; Huang, W.; Shen, H.; Zhao, X.; Cheng, L.; Wang, M.; Zhang, L. Bioorg. Med. Chem. Lett. 2014, 24, 983.

[12] Gabr, M. T.; El-Gohary, N. S.; El-Bendary, E. R.; El-Kerdawy, M. M.; Ni, N. Chin. Chem. Lett. 2016, 27, 380.

[13] Nartop, D.; Özkan, E. H.; Gündem, M.; Çeker, S.; Ağar, G.; Öğütcü, H.; Sarı, N. J. Mol. Struct. 2019, 1195, 877.

[14] Rakesh, K. P.; Kumara, H. K.; Ullas, B. J.; Shivakumara, J.; Channe Gowda, D. Bioorg. Chem. 2019, 90, 103093.

[15] Alafeefy, A. M.; Bakht, M. A.; Ganaie, M. A.; Ansarie, M. N.; El-Sayed, N. N.; Awaad, A. S. Bioorg. Med. Chem. Lett. 2015, 25 , 179.

[16] Wang, Y. Y.; Xu, F. Z.; Zhu, Y. Y.; Song, B.; Luo, D.; Yu, G.; Chen, S.; Xue, W.; Wu, J. Bioorg. Med. Chem. Lett. 2018, 28, 2979.

[17] Kumar, K. S.; Ganguly, S.; Veerasamy, R.; De Clercq, E. Eur. J. Med. Chem. 2010, 45, 5474 .

[18] Saadaoui, I.; Krichen, F.; Ben Salah, B.; Ben Mansour, R.; Miled, N.; Bougatef, A.; Kossentini, M. J. Mol. Struct. 2019, 1180, 344.

[19] Yahaya, I.; Chemchem, M.; Aydıner, B.; Seferoğlu, N.; Erva Tepe, F.; Açık, L.; Aytuna Çerçi, N.; Türk, M.; Seferoğlu, Z. J. Photochem. Photobiol., A 2019, 368, 296.

[20] Peng, X.; Liu, P.; Pang, B.; Yao, Y.; Wang, J.; Zhang, K. Carbohydr. Polym. 2019, 216, 113 .

[21] Abdel-Mohsen, H. T.; Conrad, J.; Harms, K.; Nohr, D.; Beifuss, U. RSC Adv. 2017, 7, 17427

[22] Minn, K.; Braun, P.; Sachse, B.; Wicke, H. DE 4029648, 1992.

(Cheng, F.) 